necessary for the generation of suppressor $\mathrm{T}$ cells.

Plasma from patients with SLE was added to either intact or desialized $\mathrm{T}$ cells, counterstained with fluorescein-tagged antihuman IgM, and analysed by indirect immunofluorescence. Reactivity of normal $\mathrm{T}$ cells to IgM anti-T cell antibodies from patients with SLE was markedly enhanced by their treatment with neuraminidase (Table 1). Prior adsorption of the plasma that contained antibodies to both intact and desialized $\mathrm{T}$ cells with intact $\mathrm{T}$ cells could completely eliminate the ability of plasma to bind to intact $\mathrm{T}$ cells, but only slightly to desialized $\mathrm{T}$ cells. The ability of the plasma to bind to either intact or desialized $\mathrm{T}$ cells was entirely removed by prior adsorption with desialized $\mathrm{T}$ cells. These results indicate that SLE anti-T cell antibodies consist of two antibodies with different target cell specificities; one directed at only desialized $\mathrm{T}$ cells and the other directed at both intact and desialized $\mathrm{T}$ cells.

When patients with SLE were studied simultaneously with regard to Con A-induced suppressor cell activity produced by their own $T$ cells and with regard to the presence of antibodies to either intact or desialized $\mathrm{T}$ cells in their plasma, suppres- sor $\mathrm{T}$ cell activity observed in lymphocytes of SLE patients correlated well inversely with the presence of IgM, but not IgG, antibodies directed at desialized, but not intact, $\mathrm{T}$ cells (Fig 1). These results suggest that $\operatorname{IgM}$ antibodies to desialized, rather than intact, $\mathrm{T}$ cells are responsible for the preferential elimination of suppressor $\mathrm{T}$ cells. Indeed, prior treatment of desialized, but not intact, $\mathrm{T}$ cells obtained from normals with SLE plasma which contained antibodies directed at desialized, but not intact, $\mathrm{T}$ cells, plus complement prevented the generation of Con A-induced suppressor cells.

We further demonstrated that $\mathrm{D}$-(+)lactose markedly inhibited the binding of SLE plasma to desialized $\mathrm{T}$ cells, but only slightly to intact $\mathrm{T}$ cells, and that reactivity of desialized $\mathrm{T}$ cells to SLE plasma was markedly inhibited by their treatment with proteolytic enzyme, pronase. These results suggest that antibodies directed at desialized $\mathrm{T}$ cells recognize terminal asialo-glyco $(\beta$ galactosyl residue) protein on the cell surface. In conclusion, the loss of suppressor $\mathrm{T}$ cells in patients with SLE may be the result of the effects of the activity of anti-T cell antibodies, especially antibodies to desialized $\mathrm{T}$ cells in vivo.

\title{
(3) Lymphocyte Function in autoimmune Disease
}

\author{
Kiyoaki Tanimoto, MD \\ Department of Internal Medicine and Physical Therapy, \\ Faculty of Medicine, University of Tokyo
}

Disturbed lymphocyte function is the most plausible candidate for the development of systemic autoimmune disease such as systemic lupus erythematosus (SLE) and rheumatoid arthritis (RA). Recently primary polyclonal B-cell activation has been reported to be an initial immunological event in spontaneously occurring autoimmune mice such as NZB/W Fl, MRL/l, and BXSB mice although loss of suppressor T-cells has been well established in these mice as well as in human SLE.

In the present study in vitro lymphocyte functions were studied in autoimmune dis- 
eases and the effect of humoral factors especially of antilymphocyte antibody (ALA) was observed in relation to the lymphocyte function.

Peripheral blood lymphocytes (PBL) were isolated from patients with SLE and in vitro immunoglobulin (Ig) synthesis by them was observed in the presence or absence of pokeweed mitogen (PWM). In the presence of PWM, PBL of SLE showed suppressed Ig synthesis whereas in the absence of PWM they were more significantly enhanced than

PWM - induced Ig synthesis in normal and SLE PBL ( $1 \times 10^{5}$ cells)

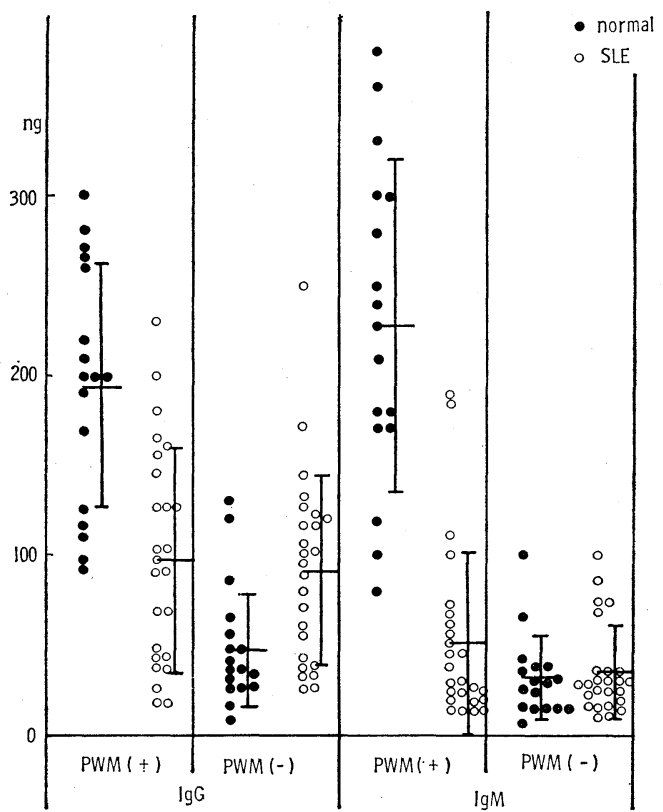

Fig. 1. In vitro Ig synthesis in normal and SLE-PBL. One million $\left(1 \times 10^{6}\right)$ of PBL from 25 patients with SLE and 17 normal healthy donors were cultured with $10 \mu \mathrm{g} / \mathrm{ml}$ of PWM and the levels of $\operatorname{IgG}$ and $\operatorname{IgM}$ were measured in the culture supernatants. The resulte were compared with those in the absence of PWM. IgG production was suppressed in SLE-PBL in the presence of PWM $(p<0.001)$, whereas in the absence of PWM IgG synthesis was enhanced in SLE-PBL $(\mathrm{p}<0.05)$. IgM production was also suppressed in SLE-PBL in the presence of PWM $(\mathrm{p}<0.00001)$ while in the absence of PWM it was not significantly different from normal controls $(p>0.05)$. The values were expressed as ng per $100 \mu \mathrm{l}$ of $1 \times 10^{6}$ cells $/ \mathrm{ml}$. normal PBL (Figure 1). Co-culture study of the combinations of T-rich and B-rich fractions suggested that reduced Ig synthesis in the presence of PWM was ascribed to the defects of both $\mathrm{T}$ and $\mathrm{B}$ cells of SLE PBL while raised synthesis in the absence of PWM was primarily due to the hyperreactivity of B-cells (Figure 2). Time course study of in vitro Ig synthesis indicated that PBL from SLE patients produced IgG as early as first 24 hours in the absence of PWM. These results suggested that B-cells from PBL of SLE were already activated in vivo by unknown substances and resulted in spontaneous IgG production without the aid of T-cells. Similar results were obtained in the study of in vitro Ig synthesis by rheumatoid synovial fluid lymphocytes. Again hyporeactivity against PWM and spontaneous B-cell activation was demonstrated. The effects of cell-free rheumatoid synovial fluids were also observed on in

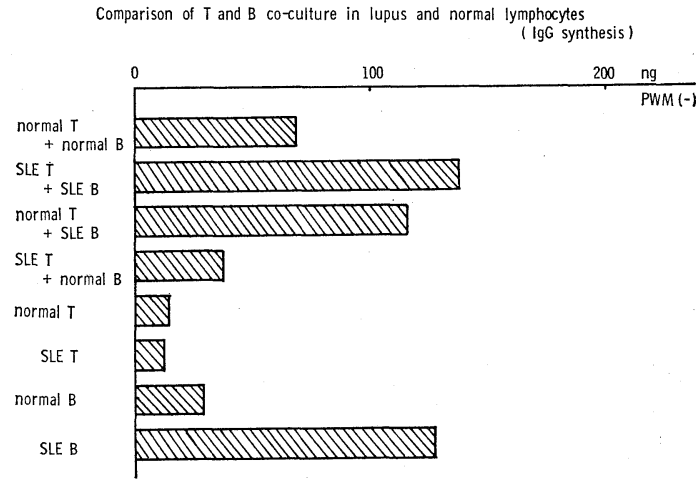

Fig. 2. Effect of $\mathrm{T}$ - and B-cell coculture "on in vitro $\mathrm{Ig}$ synthesis in normal and lupus $\mathrm{PBL}$ in the absence of PWM. Coculture experiments were performed in the absence of PWM. In the absence of PWM IgG production was reduced to less than $20 \%$ of PWM-induced IgG production in the combination of normal $\mathrm{T}$ and normal $\mathrm{B}$ cells. On the other hand, the combination of SLE B cells with normal or SLE T cells or independent culture of SLE B cells alone resulted in more pronounced IgG synthesis than the combination of normal $\mathrm{T}$ and $\mathrm{B}$ cells, while other combinations such as SLE $\mathrm{T}$ and normal $\mathrm{B}$ cells, SLE $T$ cells alone, normal $T$ cells alone, and normal $\mathrm{B}$ cells alone did not produce significant amount of $\operatorname{IgG}$. 
vitro IgG synthesis from normal PBL. The synovial fluids inhibited in vitro IgG synthesis of normal PBL in the presence of PWM, whereas they enhanced in vitro IgG synthesis in the absence of PWM. The enhancement of IgG synthesis was primarily ascribed to the activation of $\mathrm{T}$-cells by the synovial fluids in the absence of PWM while primary cause for the reduction of IgG synthesis was unclear in the presence of PWM.

The specificity of ALA was determined against IgG Fc receptor bearing T-lymphocytes $(\mathrm{T} \gamma$-cells) and non-bearing cells $(\mathrm{T} \gamma(-)$ cells). The reactivity of ALA was divided into $\mathrm{T} \gamma$ dominant type, $\mathrm{T} \gamma(-)$ dominant type and non selective type and Ig classes of ALA were determined at the same time (Table 1). $\mathrm{T} \gamma$ dominant type was mainly reactive with suppressor $\mathrm{T}$-cells and $\mathrm{T} \gamma(-)$ dominant type was reactive with helper T-cells. The cytotoxic activities of ALA against $\mathrm{T} \gamma$ or $\mathrm{T} \gamma(-)$ cells were significantly higher than those against B-cells. Immunoglobulin classes of ALA were also determined by anti Ig Sepharose treatment, Con ASepharose treatment or protein A-Sepharose treatment of the sera. IgG and IgM classes were equally distributed in $\mathrm{T} \gamma$ specific ALA while IgM type was predominant in $\mathrm{T} \gamma(-)$ specific ALA. Among these several types of ALA, IgG type of $\mathrm{T} \gamma$ specific ALA was most closely correlated with clinical disease

Table 1. Specificities of anti-lymphocyte antibodies against $\mathrm{T}$ cell subsets

\begin{tabular}{|c|c|c|}
\hline Tr dominant ${ }^{*}$ & $21.3 \%$ & $(51 / 240)$ \\
\hline Tr $(-)$ dominant & $27.9 \%$ & $(67 / 240)$ \\
\hline no difference & $50.8 \%$ & $(122 / 240)$ \\
\hline
\end{tabular}

*The cytotoxic activities against $\mathrm{T} \gamma$ cells and $\mathrm{T} \gamma(-)$ cells in the individual serum were compared each other. More than 20\% of the difference were considered significant, i. e., T $\gamma$ dominant type showed more than $20 \%$ of the cytotoxicities against $\mathrm{T} \gamma$ cells than against $\mathrm{T} \gamma(-)$ cells, and $\mathrm{T} \gamma(-)$ dominant type vice versa. No difference means less than 20\% of the difference in the cytotoxicities against $\mathrm{T} \gamma$ and $\mathrm{T} \gamma(-)$ cells. activity and laboratory findings such as anti DNA activity, serum complement levels and circulating immune complex levels. In addition, the shift of both $\mathrm{T} \gamma$ and $\mathrm{T} \gamma(-)$ specific ALA from IgM to IgG class was observed from inactive phase to active phase in a typical SLE patient after massive corticosteroid therapy although the shift of $\mathrm{Ig}$ classes was more conspicuous in $\mathrm{T} \gamma$ specific ALA than in $\mathrm{T} \gamma(-)$ specific ALA.

Natural killer (NK) activities were measured in PBL from patients with SLE, RA and Sjögren's syndrome (SjS). Reduced NK activities were demonstrated in SLE and SjS. The reduced NK activities did not correlate with clinical disease activity, nor with dose of prednisolone. When the sera from patients with SLE and $\mathrm{SjS}$ were incubated with normal PBL in the presence of rabbit complement, the former considerably inhibited NK activities but the inhibition by the latter did not reach a significant level, suggesting SLA sera contained anti NK activities, but $\mathrm{SjS}$ sera did not. In patients with RA, NK activities were normal as a whole but elevated only in patients receiving gold therapy. Gold sodium thiomalate enhanced NK activities in in vitro studies. The results of NK activities were compared with cell-mediated lympholysis. As already mentioned, ALA contained in SLE sera inhibited NK activities while they did not affect cytotoxic T-cells induced by allogeneic cells. The results indicated that NK cells are different population from cytotoxic $\mathrm{T}$ cells and ALA of SLE sera usually contain anti NK activity but did not anticytotoxic T-cell activity.

In conclusion, both $\mathrm{T}$ - and B-cell disturbances were demonstrated in autoimmune diseases. However, it has not been determined yet whether a primary cause of the development of autoimmunity exists in $\mathrm{T}$ cell defects or B-cell defects. Humoral factors such as antilymphocyte antibody may participate in these disturbances and may promote the pathologic change. 